\title{
Plasticizer-free polymer membrane potentiometric sensors based on molecularly imprinted polymers for determination of neutral phenols
}

\author{
Kaikai Liu ${ }^{\text {a, b, } 1 \text {, Yuehai Song }}{ }^{\text {b, } 1}$, Dean Song ${ }^{\text {, ** }}$, Rongning Liang a, d, * \\ ${ }^{a}$ CAS Key Laboratory of Coastal Environmental Processes and Ecological Remediation, Yantai Institute of Coastal Zone Research (YIC), Chinese Academy of \\ Sciences (CAS), Shandong Key Laboratory of Coastal Environmental Processes, YICCAS, Yantai, Shandong, 264003, PR China \\ ${ }^{\mathrm{b}}$ School of Environmental and Material Engineering, Yantai University, Yantai, Shandong, 264005, PR China \\ ${ }^{c}$ The State Agriculture Ministry Laboratory of Quality \& Safety Risk Assessment for Tobacco, Tobacco Research Institute, Chinese Academy of Agricultural \\ Sciences, Qingdao, 266101, PR China \\ ${ }^{\mathrm{d}}$ Center for Ocean Mega-Science, Chinese Academy of Sciences, Qingdao, Shandong, 266071, PR China
}

\section{H I G H L I G H T S}

- A novel plasticizer-free MIP-based potentiometric sensor is developed.

- A new copolymer, MMA-2-EHA, is synthesized and used as the sensing membrane matrix.

- The proposed MIP sensor shows an excellent sensitivity and a good selectivity.

- The proposed sensor exhibits obvious advantages in cytotoxicity and hydrophobicity.

- The copolymer membrane-based strategy is generally applicable for chemical sensors.

\section{A R T I C L E I N F O}

Article history:

Received 9 March 2020

Received in revised form

22 April 2020

Accepted 28 April 2020

Available online 30 April 2020

\section{Keywords:}

Ion-selective electrode

Molecularly imprinted polymer

Plasticizer-free membrane

\section{G R A P H I C A L A B S T R A C T}

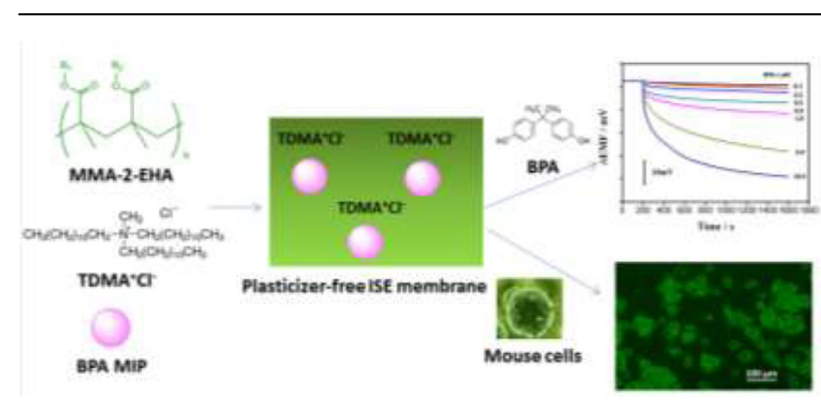

\section{A B S T R A C T}

Polymeric membrane potentiometric sensors based on molecularly imprinted polymers (MIPs) as the receptors have been successfully developed for detection of organic and biological species. However, it should be noted that all of the polymeric membrane matrices of these sensors developed so far are the plasticized poly(vinyl chloride) (PVC) membranes, which are usually suffered from undesired plasticizer leaching. Hence, for the first time, we describe a novel plasticizer-free MIP-based potentiometric sensor. A new copolymer, methyl methacrylate and 2-ethylhexyl acrylate (MMA-2-EHA), is synthesized and used as the sensing membrane matrix. By using neutral bisphenol A (BPA) as a model, the proposed plasticizer-free MIP sensor shows an excellent sensitivity and a good selectivity with a detection limit of $32 \mathrm{nM}$. Additionally, the proposed MMA-2-EHA-based MIP membrane exhibits lower cytotoxicity, higher hydrophobicity and better MIP dispersion ability compared to the classical plasticized PVC-based MIP sensing membrane. We believed that the new copolymer membrane-based MIP sensor can provide an

\footnotetext{
* Corresponding author. CAS Key Laboratory of Coastal Environmental Processes and Ecological Remediation, Yantai Institute of Coastal Zone Research (YIC), Chinese Academy of Sciences (CAS), Shandong Key Laboratory of Coastal Environmental Processes, YICCAS, Yantai, Shandong, 264003, PR China. Tel.: +86 535 2109234; fax: +865352109000.

** Corresponding author. Tel.: +86 535 2109234; fax: +86 5352109000.

E-mail addresses: songdean07@mails.ucas.ac.cn (D. Song), rnliang@yic.ac.cn (R. Liang).

1 Authors contributed equally to this work.
} 
appealing substitute for the traditional PVC membrane sensor in the development of polymeric membrane-based electrochemical and optical MIP sensors.

2020 Elsevier B.V. All rights reserved.

\section{Introduction}

Nowadays, ionophore-based polymeric membrane ion-selective electrodes (ISEs) have been widely employed as a powerful tool for determination of ionic species in clinical and environmental applications [1-5]. Especially in clinical analysis, such sensors have become the standard technique for detection of blood electrolytes as well as for measuring the $\mathrm{pH}$ value. It can be safely estimated that each year over a billion measurements with the ISEs are preformed globally in clinical laboratories alone. In particular, recent improvements in the detection limits of these sensors yielded sensors for the direct measurement in the subnanomolar concentration range [6,7]. However, note that, in these applications, most of the targets are inorganic ions such as blood electrolyte ions and heavy metal ions. This is due to the lack of the receptors (ionophores) for selective recognition of other species such as organic and biological species. So far, the synthetic receptors for selective recognition of organic and biological species are still rather rare.

Molecularly imprinted polymers (MIPs) have been regarded as general materials for selective recognition of a wide range of analytes with affinities and selectivities comparable to natural receptors such as antibodies and enzyme [8,9]. Especially, MIPs are stable, less costly and easier to produce. These characteristics make these receptors particularly suitable for their use as the receptors of the ISEs for selective recognition of organic and biological species. Nowadays, MIP-based polymeric membrane potentiometric sensors have been successfully used for potentiometric detection of organic ions [10,11], neutral organic species [12-17] and biological species [18] related with clinical and environmental applications. These spectacular advances have facilitated new applications for which potentiometric sensors have not been used traditionally. For MIP-based potentiometric sensors, a typical membrane composition is $31 \mathrm{wt} \%$ polymer matrix (usually poly(vinyl chloride), PVC), $62 \mathrm{wt} \%$ plasticizer, $6 \mathrm{wt} \%$ MIP receptors and a small amount of membrane additives. However, like the classical ISEs, there are many disadvantages associated with the usage of the PVC matrix [19-22]. One of the most significant disadvantages is the plasticizer leaching from the sensing membranes, which can cause decreased sensor lifetimes, unstable potential responses and increased sample perturbation. Moreover, for in-vivo measurements, where biocompatibility is essential, the plasticizer leaching may induce a serious inflammatory response in the vicinity of the electrode because the plasticizer is often toxic. Other disadvantages of the plasticizer usage are related to the sensor miniaturization, in which the use of plasticizers has been shown to be detrimental to the fabrication processes due to their poor adhesion to the electrode substrates.

For classical ISEs, several strategies have been proposed to overcome these disadvantages, including grafting of the membrane compositions [20,23,24], reduced use of the plasticizer [25] and use of the plasticizer-free polymer matrices [19-22]. Unfortunately, no one has been used to resolve the above-mentioned problems of the plasticized PVC membrane potentiometric sensors based on the MIPs as the receptors. This poses serious limits to their wide use of the MIP-based sensors, especially in fabrication of the miniaturized sensors and in-vivo measurements. Among these strategies developed so far, composition grafting is usually involved in complicated synthesis procedures, and reduced plasticizer content can cause high impedance of the sensing membrane. Hence, the plasticizerfree polyacrylate membrane seems to be the most promising alternative of the plasticized PVC membrane. Typically, a monomer that gives polymers with a high glass transition temperature ( $\mathrm{Tg}$ ), is reacted in an appropriate ratio with a low-Tg co-monomer to produce a copolymer with the desired physical and mechanical properties [20]. At present, such membrane has become a convenient means for producing plasticizer-free matrixes for use in ISEs due to its attractive features including easy synthesis, excellent selectivity and high water-repellent property.

Herein, we present a plasticizer-free potentiometric sensor based on the MIP for the first time. A novel polyacrylate copolymer which is composed of the monomer methyl methacrylate (MMA) with a $\mathrm{Tg}$ of $105{ }^{\circ} \mathrm{C}$ and the co-monomer 2-ethylhexyl acrylate (2EHA) with a $\mathrm{Tg}$ of $-70^{\circ} \mathrm{C}$ is used as the membrane matrix of the ISE membrane. The basic characteristics of this new copolymer MMA2-EHA in potentiometric sensing platforms in terms of response range, selectivity, cytotoxicity and hydrophobicity are described. Bisphenol A (BPA), an endocrine-disrupting chemical which can be found in the urine of the majority of people worldwide is used as a model. It will be shown that the proposed potentiometric sensor can offer a sensitive, selective and biocompatible way to detect BPA. The results may encourage further development of ultraminiaturized and low-detection-limit sensing systems.

\section{Experimental}

\subsection{Reagents and materials}

Methyl methacrylate (MMA), 2-ethylhexyl acrylate (2-EHA), nbutyl acrylate (nBA), single-walled carbon nanotubes (SWCNTs), bisphenol A (BPA), methanol, acetonitrile (ACN), PVC, o-nitrophenyl octyl ether (o-NPOE), benzene, tridodecylmethylammonium chloride (TDMAC) and tetradodecylammonium tetrakis(4chlorophenyl)borate (ETH 500) were purchased from Sigma. Methacrylic acid (MAA), divinylbenzene 80 (DVB 80), 2,2'-azobi$\mathrm{s}$ (isobutyronitrile) (AIBN), acetic acid, tetrahydrofuran (THF), phenol, catechol, 3-nitrophenol and dichloromethane (DCM) were obtained from Fluka. All other reagents were analytical grade and used without any further purification. Aqueous solutions were prepared with freshly deionized water $(18.2 \mathrm{M} \Omega \mathrm{cm}$ specific resistance) obtained with a Pall Cascada laboratory water system.

\subsection{Synthesis of the BPA MIP}

The synthesis of the MIP was according to our previous report with appropriate modifications [12]. Briefly, the template BPA (0.5 mmol), MAA (2 mmol), DVB 80 (10 $\mathrm{mmol})$, and free-radical initiator AIBN $(25 \mathrm{mg})$ were dissolved in $\mathrm{ACN}(12.5 \mathrm{~mL})$ in a $50 \mathrm{~mL}$ flask. The mixture was sonicated for $5 \mathrm{~min}$ to maintain homogeneity. Then the solution was purged with a gentle flow of $\mathrm{N}_{2}$ for $15 \mathrm{~min}$ and sealed under $\mathrm{N}_{2}$ atmosphere. Polymerization was carried out by submerging the flask in an oil bath at $80^{\circ} \mathrm{C}$ for $24 \mathrm{~h}$. After polymerization, the template was removed by batch-mode solvent extraction with methanol/acetic acid $(8 / 2, \mathrm{v} / \mathrm{v})$ and methanol. The resulting polymer was dried in vacuum overnight at $40^{\circ} \mathrm{C}$. 


\subsection{Synthesis of the plasticizer-free copolymer}

The copolymer was synthesized by the free-radical polymerization. The synthesis procedures are similar to those of the previously reported work with appropriate modifications [19]. The schematic representation of the synthesis process is shown in Fig. 1. Briefly, the monomer MMA (10 mmol), the co-monomer 2-EHA $(63 \mathrm{mmol})$ and free-radical initiator AIBN $(1 \mathrm{mmol})$ were firstly dissolved into $25 \mathrm{~mL}$ dry benzene. The solution was then degassed for $15 \mathrm{~min}$ and heated to $80^{\circ} \mathrm{C}$ and maintained at this temperature for $8 \mathrm{~h}$. After polymerization, methanol, approximately 10 times by volume, was added into the cooled polymer solution with vigorous stirring. The transparent gelatinous precipitates formed were filtered and dried under vacuum for 24 , and finally redissolved in $60 \mathrm{~mL}$ DCM. The copolymer MMA-nBA was synthesized by the similar procedure except that the co-monomer was replaced with nBA.

\subsection{Preparation of the sensing membranes and the electrodes}

Glassy carbon electrode (GCE) was firstly polished with $0.05 \mu \mathrm{m}$ alumina slurries, and then rinsed with deionized water. After being cleaned by acetone, alcohol and deionized water successively, the obtained GCE was used as the electrode substrate. For preparation of the proposed solid contact, $10 \mathrm{mg}$ of SWCNTs were dispersed in $2 \mathrm{~mL}$ THF by sonication for $2 \mathrm{~h}$, and then $50 \mu \mathrm{L}$ of the obtained mixture was drop-cast onto the above polished GCE surface and dried by an infrared lamp.

The plasticizer-free MIP membrane contained $3 \mathrm{~mL}$ of copolymer MMA-2-EHA (91.0 wt\%) dissolved in DCM, $10.55 \mathrm{mg}$ of MIP (6.0 wt\%), $1.75 \mathrm{mg}$ of TDMAC (1.0 wt\%) and $3.52 \mathrm{mg}$ of ETH 500 (2.0 wt\%). For comparison, the traditional MIP membranes contained (totaling $360 \mathrm{mg}$ ) $6.0 \mathrm{wt} \% \mathrm{MIP}, 1.0 \mathrm{wt} \%$ TDMAC, $2.0 \mathrm{wt} \% \mathrm{ETH}$ $500,36.4 \mathrm{wt} \%$ PVC and $54.6 \mathrm{wt} \%$ o-NPOE were dissolved in $3.6 \mathrm{~mL}$ THF. $90 \mu \mathrm{L}$ of the membrane cocktail was drop-cast on to the transducer layer and allowed to dry for $6 \mathrm{~h}$. Before measurements, all the electrodes were conditioned overnight in $33 \mathrm{mM}$ of $\mathrm{pH} 7.5$ phosphate buffer solution (PBS). The electromotive force (EMF) measurements were performed at room temperature by using a CHI 760C electrochemical workstation (Shanghai Chenhua Apparatus Corporation, China) with $\mathrm{Ag} / \mathrm{AgCl}(3 \mathrm{M} \mathrm{KCl})$ as the reference electrode. The surface hydrophilicity was evaluated by water contact angle measurements using the sessile drop method recorded by an optical instrument (ADS300, Data Physics, Germany).

\subsection{Cell culture and cytotoxicity test}

The mouse macrophage cells, RAW 264.7, were kindly provided by the Medicinal Chemistry Lab of Yantai University (Yantai, China). The cells were maintained in complete RPMI 1640 medium supplemented with $10 \%$ heat-inactivated fetal bovine serum, $1 \% \mathrm{~L}$ glutamine, $1 \%$ penicillin and streptomycin, and $1.5 \%$ sodium bicarbonate at $37{ }^{\circ} \mathrm{C}$ in a humidified $5 \% \mathrm{CO}_{2}$ atmosphere.

A volume of $90 \mu \mathrm{L}$ of the corresponding membrane cocktail was drop-casted on the bottom part of the sterile petri dish and left to dry for $24 \mathrm{~h}$ until complete evaporation of the solvent. The dish containing the membrane was sterilized for 15 min under a UV lamp. Then, $10 \mathrm{ml}$ of the cultured cell suspension was added to the sterile petri dish and left to grow at $37^{\circ} \mathrm{C}$ for $24 \mathrm{~h}$. The cytotoxicity of the proposed MIP-based plasticizer-free polymeric membrane was evaluated by using the confocal laser scanning microscopy (CLSM) analysis (Fluo View FV 1000, Olympus, Japan). The MTT assays were carried out as described by Mosmann [26] to further evaluate the cytotoxicity by measuring the cell viability. The comparison experiments were performed in parallel for the traditional MIP-based PVC polymeric membrane. All apparatus used in this study were autoclaved prior to the biological experiments, and the operations were carried out in a biosafety cabinet.

\section{Results and discussion}

During the past few decades, tremendous advances have been made in MIP-based potentiometric sensors. Nowadays, MIP-based potentiometric sensors have been successfully used for potentiometric detection of organic ions, neutral organic species and biological species related with clinical and environmental applications

\section{MMA 2-EHA}<smiles>C=C(C)C(=O)OCC(CC)CCCC</smiles>

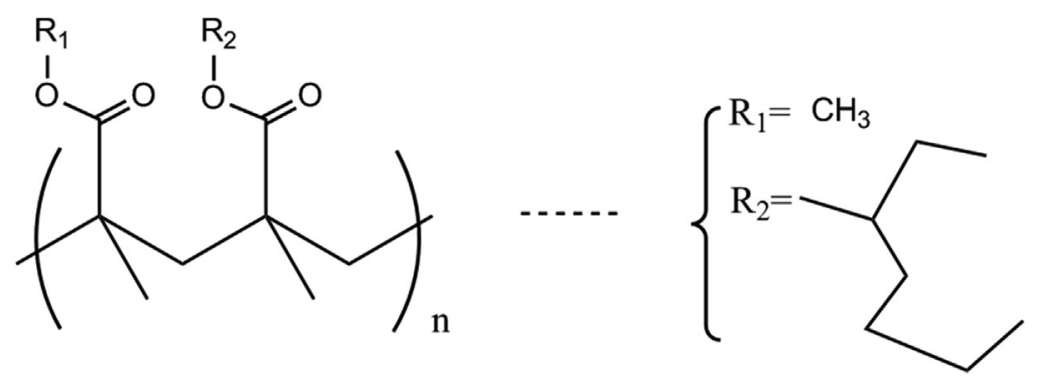

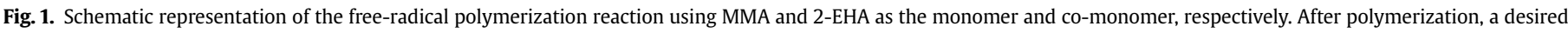
non-crosslinked plasticizer-free ion-selective membrane matrix material can be obtained. 


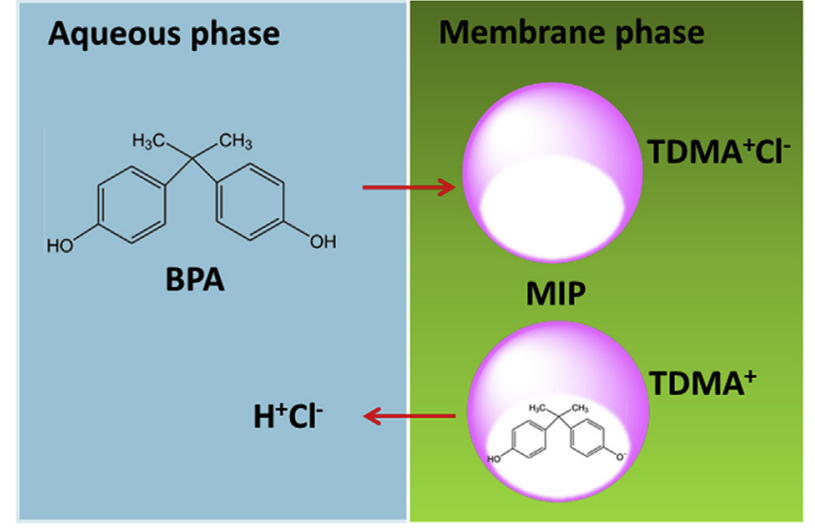

Scheme 1. Representation of the possible mechanism of the proposed sensor for potentiometric detection of BPA.

[10-18]. These spectacular advances have facilitated new applications for which polymeric membrane potentiometric sensors have not been used traditionally. However, note that, all of the matrices of these MIP-based sensors are the plasticized membrane. The presence of the plasticizers may cause some problems such as plasticizer leaching, inflammatory response and weak adhesion ability. Such problems limit their wide use of these sensors, especially in in-vivo and miniaturized sensor applications. Hence, to overcome these problems, in this work, a plasticizer-free MIPbased polymeric membrane potentiometric sensor is described using a new MMA-2-EHA copolymer as a matrix. The new concept is evaluated for potentiometric detection of BPA. The representation of the possible mechanism of the proposed sensor for potentiometric detection of BPA is illustrated in Scheme 1. The response principle is analogous to that of the potentiometric neutral phenol sensors described earlier [27]. When the electrode is in contact with the sample solution containing neutral BPA, BPA can be favorably extracted from the aqueous phase into the membrane phase through the selective interactions between BPA and the MIP in the membrane, which leads to the proton dissociation of the complexed BPA. The dissociated proton can associate the counter ion of the ion exchanger (e.g., $\mathrm{Cl}^{-}$of TDMAC) in the membrane and then diffuse into the aqueous phase. This process facilitates the net movement of anionic species from the membrane phase to the aqueous phase and thus induces an anionic potential response.

\subsection{Optimization of the membrane composition}

Previous studies have demonstrated that electrically neutral phenols and their derivatives could generate strong anionic potential responses on quaternary ammonium salt-doped polymeric membranes under near-neutral $\mathrm{pH}$ conditions. These unexpected anionic responses can be attributed to the net movement of hydrogen ions from the membrane phase to the aqueous phase stimulated by neutral phenols [27]. Additionally, in our previous work, it has been found that the potential responses to dissociated phenols are much lower than those to neutral phenols [14]. Therefore, we explored the application of the proposed sensor in detection of neutral BPA. In order to ensure that BPA exists all in their neutral forms, $33 \mathrm{mM}$ PBS buffer of $\mathrm{pH} 7.5$ was used as the background medium.

Like the classical plasticized polymeric membrane ISEs, the membrane composition of plasticizer-free MIP sensor also has a profound impact on the sensing performance of the polymeric membrane ISE. Therefore, the influence of membrane composition on the potential response was tested and the results are shown in
Figs. S1 and S2 in the Supporting Information.

It has been well established that the sensitivity of the membrane electrode depends on the amount of the MIP which determines the number of the binding sites [10]. Therefore, the effect of the MIP content on the sensitivity of the electrode was examined (Fig. S1). It can be seen that the potential change increases upon increasing the amount of the MIP, which is probably owing to the increase in the number of the binding sites for molecular recognition. However, at amounts above $6.0 \mathrm{wt} \%$, MIP may not be well dispersed in the sensing membrane, which can cause high membrane impedance and thus worsen the detection limit. Therefore, the MIP amount of $6.0 \mathrm{wt} \%$ with a maximum response was chosen for the proposed sensor.

The influence of the copolymer matrix on the sensor response was investigated. Two kinds of plasticizer-free ISEs based on the MIPs were firstly prepared: one with MMA-2-EHA as the matrix and the other with MMA-nBA. For measurement of BPA at $1 \mu \mathrm{M}$, the EMF change obtained by the MMA-2-EHA-based sensor is approximately $15 \mathrm{mV}$, while that by the electrode with the MMAnBA matrix is $5 \mathrm{mV}$ (Fig. S2). This improvement in sensitivity could be probably due to the better dispersion of the BPA MIP in the MMA-2-EHA matrix than that in the MMA-nBA matrix. Therefore, the MMA-2-EHA matrix was selected as the matrix throughout the study.

\subsection{Performance of the MIP-based plasticizer-free potentiometric sensor}

Under the optimized conditions, Fig. 2 displays the typical dynamic potential responses of plasticizer-free potentiometric sensor based on the MIP receptors for measuring neutral BPA at concentrations ranging from 0.1 to $10 \mu \mathrm{M}$ in $33 \mathrm{mM}$ PBS background of $\mathrm{pH}$ 7.5. It can be seen that the proposed electrode shows the nonclassical potential responses to neutral BPA (Fig. 2a), which is consistent with the results of the previous studies. In addition, it should be noted that the proposed plasticizer-free sensor shows a longer response time than the traditional MIP-based plasticized polymeric membrane potentiometric sensors [13]. Here, the response time was defined as the time required to achieve the same EMF change at the same concentration of neutral phenols. Such longer response time might be attributed to the fact that the diffusion coefficient for the target in the plasticizer-free copolymer is smaller than that in the classical plasticized PVC membrane. According to the previous report [28], the diffusion coefficients of ions in methacrylic-acrylic acid ISE membranes are about 2 orders of magnitude lower than the corresponding values in plasticized PVC membranes, which significantly results in a longer response time. Detailed experimental results reveal that the potential difference is proportional to the concentration of neutral BPA in the concentration range of $0.1-1 \mu \mathrm{M}$ (Fig. 2b). In this work, the potential difference between the baseline and the potential measured at $1400 \mathrm{~s}$ after BPA addition was used for quantification. In this case, the calculated detection sensitivity of the proposed MIP-based electrode is $0.89 \mathrm{mV} / \mu \mathrm{M}$ and a lower detection limit of $32 \mathrm{nM}$ $(3 \sigma)$ could be obtained. Such detection limit is comparable for that obtained by the rotating electrode-based plasticized MIP sensor with a similar sensing membrane composition [15]. Since it has been well established that the nonclassical potential responses of interfering ion-conditioned polymeric membrane ISEs can be greatly improved by using the rotating electrode configuration [29], it can be demonstrated that the plasticizer-free membrane is effective for the improvement of the detection limit. In addition, a comparison of the analytical performances between the proposed sensor and other potentiometric bisphenol sensors based on the MIP receptors is listed in Table S1. As illustrated, the analytical 

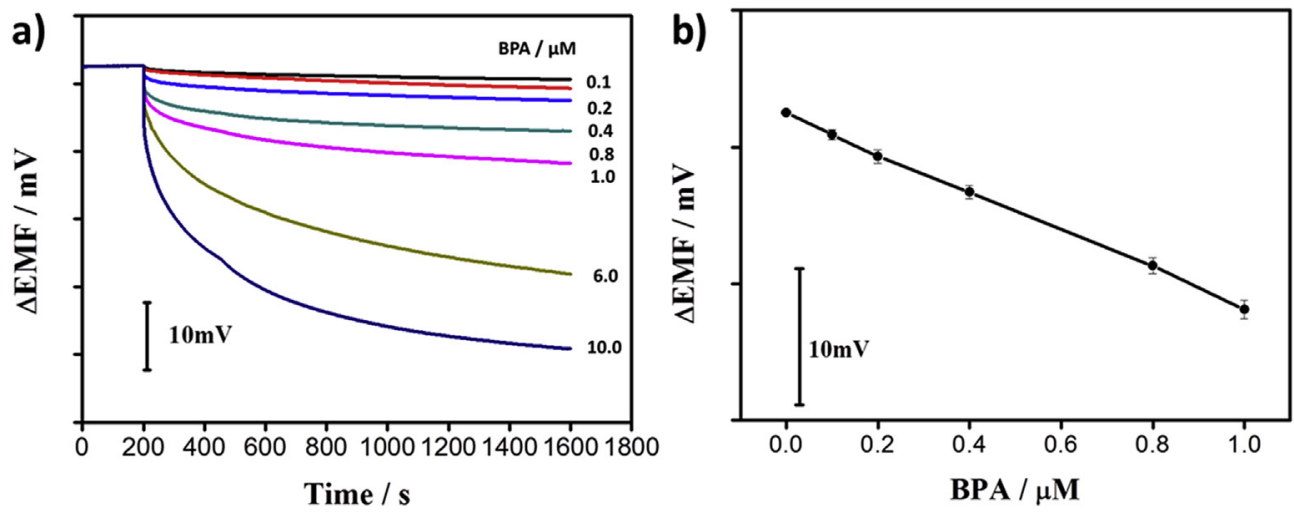

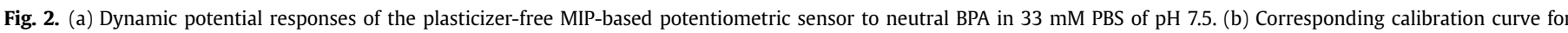

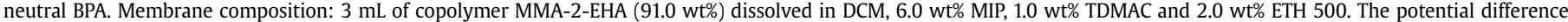

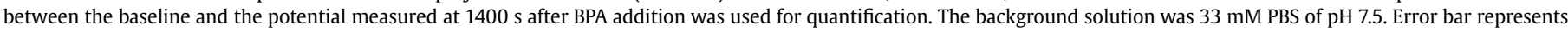
the standard deviation for one electrode with three parallel measurements.

performances of the proposed sensor are comparable with those of the previously reported potentiometric bisphenol sensors in terms of detection range and detection limit.

Reversible potentiometric detection of BPA was investigated by monitoring the potential responses of the plasticizer-free MIPbased sensor to $0.4 \mu \mathrm{M}$ BPA. After each measurement, the sensor was washed with a mixture of ethanol and $33 \mathrm{mM}$ PBS of pH 7.5 (1/ $4, v / v$ ) to remove the analyte in the MIP and then immersed in the PBS to obtain the original and reproducible baseline potential values. As illustrated in Fig. S3 in the Supporting Information, no obvious decrease in the potential response is observed after successively regeneration cycles for the electrode regenerated by the regeneration solution. Such results suggest that the MIP-based plasticizer-free polymeric membrane electrode exhibits good reversibility.

The selectivity of the proposed MIP-based plasticizer-free polymeric membrane electrode over other phenols such as phenol, catechol and 3-nitrophenol was investigated. The results are shown in Fig. 3. In order to guarantee that BPA and other phenols were in all their neutral forms, a PBS buffer with a $\mathrm{pH}$ of 7.8 was employed as the background solution for phenol and catechol while one with a $\mathrm{pH}$ of 6.3 was used for 3-nitrophenol detection. As expected, the sensor exhibits an excellent selectivity over other phenols. Compared to the responses to neutral BPA with respect to increasing the concentration from 0.1 to $1 \mu \mathrm{M}$, negligible changes in potential difference can be observed for other three phenols
(Fig. 3a), which suggests the specific recognition ability of the MIP receptor towards the target BPA. Moreover, no obvious potential changes can be found for all the phenols by using the NIP membrane-based electrode (Fig. 3b). These results indicate that the measured potential responses are induced mainly by the specific recognition interactions between the target BPA and the MIP receptor in the MMA-2-EHA membrane.

The MIP-based sensor has been found to show good reproducibility and stability for BPA detection. For $0.4 \mu \mathrm{M}$ BPA, the relative standard deviation (RSD\%) of three electrodes is $5.8 \%$. A response slope loss of $7.1 \%$ was observed after being stored at $4{ }^{\circ} \mathrm{C}$ for 1 month. Additionally, the proposed sensor was finally applied to determination of BPA in the spiked lake water samples with satisfactory results (Fig. S4 and Table S2 in the Supporting Information).

\subsection{Characterization of the plasticizer-free MIP sensing membrane}

To illustrate the low cytotoxicity and the excellent biocompatibility of the proposed plasticizer-free MIP membrane, two kinds of MIP-based ISE membranes were compared: one with the MMA-2EHA copolymer as the membrane matrix and the other with the classical PVC-o-NPOE matrix. Both membranes were firstly dropcasted onto the sterile petri dish and then left the mouse macrophage cells, RAW 264.7, to grow at $37^{\circ} \mathrm{C}$ for $24 \mathrm{~h}$. After cell proliferation, the obtained membranes were finally visualized by using the confocal laser scanning microscopy. The results are shown in
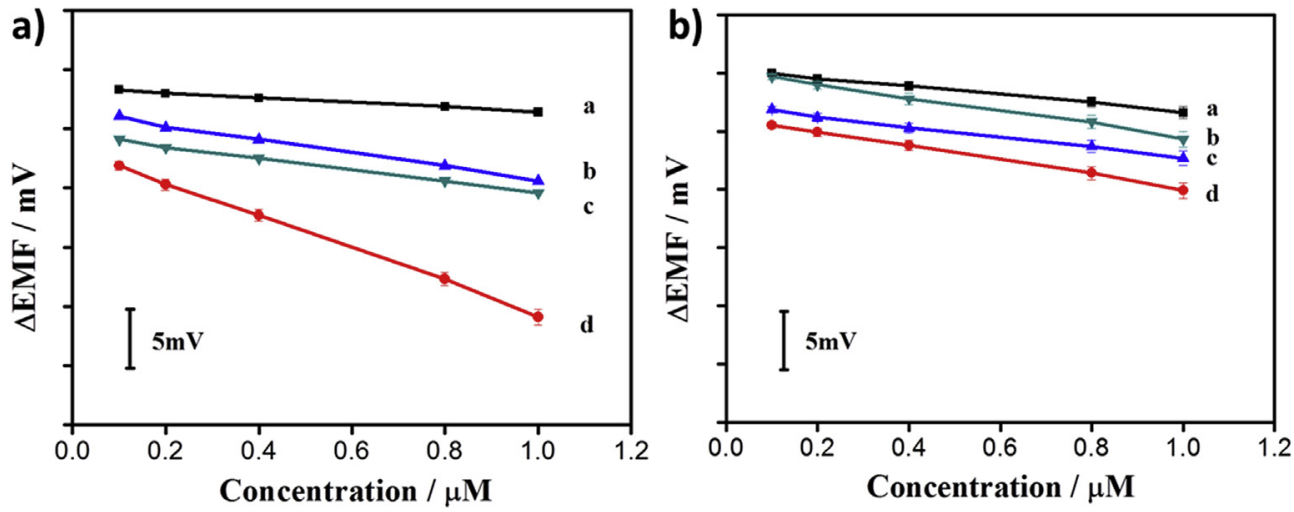

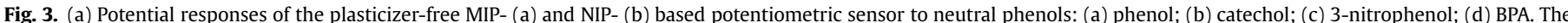

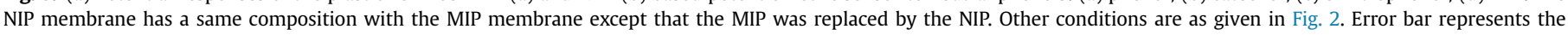
standard deviation for one electrode with three parallel measurements. 


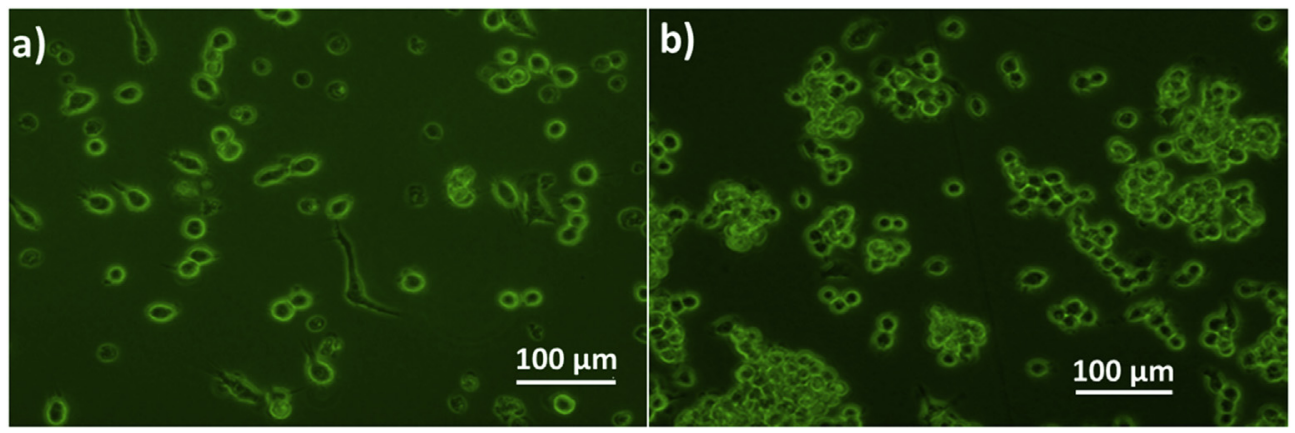

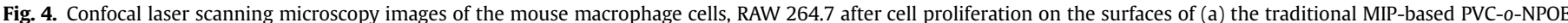

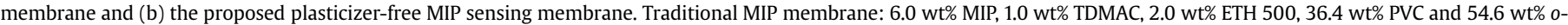
NPOE. Plasticizer-free MIP membrane: 3 mL of copolymer MMA-2-EHA (91.0 wt\%) dissolved in DCM, 6.0 wt\% MIP, 1.0 wt\% TDMAC and 2.0 wt\% ETH 500.

Fig. 4.

Clearly, the MMA-2-EHA-based MIP membrane differs obviously from the PVC-o-NPOE membrane. The classical PVC-o-NPOE sensing membrane has a small number of grown RAW 264.7 cells with densely packed cell aggregates in the visualized area (Fig. 4a), indicating that the PVC-o-NPOE matrix is potentially cytotoxic, which significantly inhibits the growth of the cells. In contrast, a significant increase in the number of the cells is observed for the MMA-2-EHA membrane (Fig. 4b). Further, the MTT assay shows that much more live cells are observed for the MMA-2-EHA-based MIP membrane as compared to the plasticized PVC-based MIP membrane (Fig. S5 in the Supporting Information). These results indicate that the proposed MMA-2-EHA-based MIP membrane shows an improved biocompatibility and a low cytotoxicity. Such excellent biocompatibility of the proposed MIP sensor offers great potential for potentiometric detection in real biological samples, especially in in-vivo potentiometric measurements.

It has been well established the use of plasticized PVC as an ionsensing membrane matrix in the absence of an ion-to-electron transducer as the solid contact has been unsuccessful in inhibiting the formation of the detrimental water layer between the electrode and the membrane [28]. Fortunately, the emergence of the hydrophobic water-repellent polyacrylate copolymer matrix is exhibiting a great potential to dramatically change this situation [30-32]. In this work, a novel polyacrylate copolymer matrix, MMA-2-EHA, was used as the matrix of the polymeric membrane ISEs. In order to illustrate the water-repellent property of such new matrix, the contact angle measurements of water droplets were performed to characterize the surface hydrophobicity of the MIPbased MMA-2-EHA membrane (Fig. 5). As a comparison, the contact angle of the MIP-based PVC-o-NPOE membrane was also tested. It can be clearly seen that the water contact angle of the copolymer membrane is larger than that of the PVC-o-NPOE membrane $\left(97.5^{\circ} \pm 2.9^{\circ}\right.$ vs $\left.82.6^{\circ} \pm 3.5^{\circ}\right)$. This result indicates that the proposed MMA-2-EHA membrane is more hydrophobic than the PVC membrane. Such characteristic makes the new membrane particularly promising for its use as a substitution of the traditional plasticized PVC membrane in the development of the solid contact ISEs.

In order to evaluate the dispersivity of the BPA MIP in the copolymer membrane matrix, the dispersion abilities of the MIP in the copolymer matrix and in the PVC matrix were compared (Fig. S6 in the Supporting Information). Experiments show that the BPA MIP can be well dispersed in the MMA-2-EHA-based matrix, and the obtained polymeric membrane is homogeneous. Such homogeneous MIP membrane could induce more binding sites available in the membrane and the lower membrane impedance. However, the MIP with a same amount cannot be dispersed well in the classical PVC membrane. Indeed, an obvious uneven surface of the PVC membrane was observed after the dispersion of the BPA MIP. Thus, it can be demonstrated that the MMA-2-EHA-based copolymer matrix is more suitable than the plasticized PVC membrane matrix for the fabrication of polymeric membrane potentiometric sensors based on the MIP as the receptors.

The MMA-2-EHA-based MIP membrane was further characterized by the scanning electron microscopy (SEM) and Fouriertransform infrared spectroscopy (FT-IR). The results are shown in Figs. S7 and S8 in the Supporting Information. As can be seen from the top-view SEM image, the plasticizer-free MIP membrane (Fig. S7) clearly exhibits a flat and homogeneous surface, which further indicates that the MIP receptors can be dispersed well in the unplasticized MMA-2-EHA matrix. In addition, the successful preparation of the MMA-2-EHA-based MIP membrane can be confirmed by the FT-IR (Fig. S8). a)

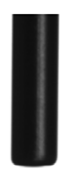

b)

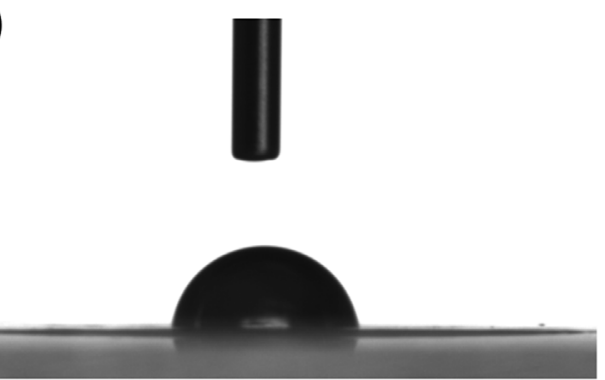

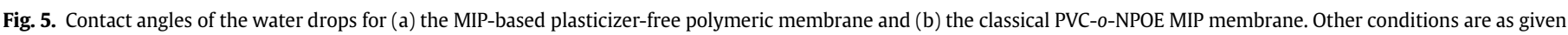
in Fig. 4. 


\section{Conclusions}

In this work, we have developed a novel plasticizer-free MIPbased potentiometric sensor for detection of neutral BPA using a new copolymer MMA-2-EHA as a membrane matrix. The sensor exhibits an excellent sensitivity towards BPA and a good selectivity over other interfering phenols, which offers a detection limit of $32 \mathrm{nM}$ for neutral BPA. In addition, the proposed MMA-2-EHAbased MIP membrane shows some obvious advantages in terms of biocompatibility, hydrophobicity and MIP dispersion ability compared to the classical plasticized PVC-based MIP sensing membrane. It can be demonstrated that the proposed MMA-2-EHA copolymer matrix would be an appealing substitute for the traditional PVC matrix in the development of polymeric membrane potentiometric sensors using the MIP as the receptors. Since many MIPs have been extensively exploited in analytical chemistry, it can be envisioned that the novel concept is promising for fabrication of various electrochemical or optical membrane sensors for sensitive, selective and biocompatible detection of a wide range of targets.

\section{Declaration of competing interest}

The authors declare that they have no known competing financial interests or personal relationships that could have appeared to influence the work reported in this paper.

\section{CRediT authorship contribution statement}

Kaikai Liu: Conceptualization, Methodology, Investigation. Yuehai Song: Conceptualization. Dean Song: Formal analysis, Writing - review \& editing. Rongning Liang: Conceptualization, Methodology, Writing - review \& editing, Supervision.

\section{Acknowledgments}

This work was financially supported by the National Natural Science Foundation of China (21874151, 41576106), the Youth Innovation Promotion Association of CAS (2014190) and the Taishan Scholar Program of Shandong Province (tspd20181215).

\section{Appendix A. Supplementary data}

Supplementary data to this article can be found online at https://doi.org/10.1016/j.aca.2020.04.074.

\section{References}

[1] E. Zdrachek, E. Bakker, Potentiometric sensing, Anal. Chem. 91 (2019) 2-26.

[2] J. Hu, A. Stein, P. Buhlmann, Rational design of all-solid-state ion-selective electrodes and reference electrodes, Trends Anal. Chem. 76 (2016) 102-114.

[3] I. Makra, A. Brajnovits, G. Jágerszki, P. Fürjes, R.E. Gyurcsányi, Potentiometric sensing of nucleic acids using chemically modified nanopores, Nanoscale 9 (2017) 739-747.

[4] N.K. Joon, N. He, T. Ruzgas, J. Bobacka, G. Lisak, PVC-based ion-selective electrodes with a silicone rubber outer coating with improved analytical performance, Anal. Chem. 91 (2019) 10524-10531.

[5] M. Parrilla, M. Cuartero, G.A. Crespo, Wearable potentiometric ion sensors, Trends Anal. Chem. 76 (2016) 102-114.

[6] T. Sokalski, A. Ceresa, T. Zwickl, E. Pretsch, Large improvement of the lower detection limit of ion-selective polymer membrane electrodes, J. Am. Chem. Soc. 119 (1997) 11347-11348.

[7] E. Bakker, E. Pretsch, Modern potentiometry, Angew. Chem. Int. Ed. 46 (2007)
$5660-5668$.

[8] K. Haupt, K. Mosbach, Molecularly imprinted polymers and their use in biomimetic sensors, Chem. Rev. 100 (2000) 2495-2504.

[9] L.X. Chen, S.F. Xu, J.H. Li, Recent advances in molecular imprinting technology: current status, challenges and highlighted applications, Chem. Soc. Rev. 40 (2011) 2922-2942.

[10] R.N. Liang, R.M. Zhang, W. Qin, Potentiometric sensor based on molecularly imprinted polymer for determination of melamine in milk, Sens. Actuators, B 141 (2009) 544-550.

[11] P.J. Li, R.N. Liang, X.F. Yang, W. Qin, Imprinted nanobead-based disposable screen-printed potentiometric sensor for highly sensitive detection of 2 naphthoic acid, Mater. Lett. 225 (2018) 138-141.

[12] R.N. Liang, D.A. Song, R.M. Zhang, W. Qin, Potentiometric sensing of neutral species based on a uniform-sized molecularly imprinted polymer as a receptor, Angew. Chem. Int. Ed. 49 (2010) 2556-2559.

[13] H. Zhang, R.Q. Yao, N. Wang, R.N. Liang, W. Qin, Soluble molecularly imprinted polymer-based potentiometric sensor for determination of bisphenol AF, Anal. Chem. 90 (2018) 657-662.

[14] T.T. Wang, R.N. Liang, T.J. Yin, R.Q. Yao, W. Qin, An all-solid-state imprinted polymer-based potentiometric sensor for determination of bisphenol S, RSC Adv. 6 (2016) 73308-73312.

[15] L.J. Kou, R.N. Liang, X.W. Wang, Y. Chen, W. Qin, Potentiometric sensor for determination of neutral bisphenol A using a molecularly imprinted polymer as a receptor, Anal. Bioanal. Chem. 405 (2013) 4931-4936.

[16] R.N. Liang, L.S. Chen, W. Qin, Potentiometric detection of chemical vapors using molecularly imprinted polymers as receptors, Sci. Rep. 5 (2015) $12462-12470$.

[17] L.B. Qi, R.N. Liang, W. Qin, Stimulus-responsive imprinted polymer-based potentiometric sensor for reversible detection of neutral phenols, Anal. Chem. 92 (2020) 4284-4291.

[18] R.N. Liang, J.W. Ding, S.S. Gao, W. Qin, Mussel-inspired surface-imprinted sensors for potentiometric label-free detection of biological species, Angew. Chem. Int. Ed. 56 (2017) 6833-6837.

[19] L.Y. Heng, E.A.H. Hall, Producing "self-plasticizing" ion-selective membranes, Anal. Chem. 72 (2000) 42-51.

[20] Y. Qin, S. Peper, A. Radu, A. Ceresa, E. Bakker, Plasticizer-free polymer containing a covalently immobilized $\mathrm{Ca}^{2+}$-selective ionophore for potentiometric and optical sensors, Anal. Chem. 75 (2003) 3038-3045.

[21] Y. Qin, S. Peper, E. Bakker, Plasticizer-free polymer membrane ion-selective electrodes containing a methacrylic copolymer matrix, Electroanalysis 14 (2002) 1375-1381.

[22] S. Peper, A. Ceresa, Y. Qin, E. Bakker, Plasticizer-free microspheres for ionophore-based sensing and extraction based on a methyl methacrylatedecyl methacrylate copolymer matrix, Anal. Chim. Acta 500 (2003) 127-136.

[23] Y. Liu, Y. Xue, H. Tang, M. Wang, Y. Qin, Click-immobilized $\mathrm{K}^{+}$-selective ionophore for potentiometric and optical sensors, Sens. Actuators, B 171-172 (2012) 556-562.

[24] T.J. Yin, J.H. Li, W. Qin, An all-solid-state polymeric membrane $\mathrm{Ca}^{2+}$-selective electrode based on hydrophobic alkyl-chain-functionalized graphene oxide, Electroanalysis 29 (2017) 821-827.

[25] E. Lindner, V.V. Cosofret, S. Ufer, R.P. Buck, W.J. Kao, M.R. Neuman, J.M. Anderson, Ion-selective membranes with low plasticizer content: electroanalytical characterization and biocompatibility studies, J. Biomed. Mater. Res. 28 (1994) 591-601.

[26] T. Mosmann, Rapid colorimetric assay for cellular growth and survival: applications to proliferation and cytotoxicity assays, J. Immunol. Methods 16 (1983) 55-63.

[27] T. Ito, H. Radecka, K. Tohda, K. Odashima, Y. Umezawa, On the mechanism of unexpected potentiometric response to neutral phenols by liquid membranes based on quaternary ammonium salts-systematic experimental and theoretical approaches, J. Am. Chem. Soc. 120 (1998) 3049-3059.

[28] J.P. Veder, R. De Marco, G. Clarke, R. Chester, A. Nelson, K. Prince, E. Pretsch, E. Bakker, Elimination of undesirable water layers in solid-contact polymeric ion-selective electrodes, Anal. Chem. 80 (2008) 6731-6740.

[29] Q.S. Ye, M.E. Meyerhoff, Rotating electrode potentiometry: lowering the detection limits of nonequilibrium polyion-sensitive membrane electrodes, Anal. Chem. 73 (2001) 332-336.

[30] A. Michalska, M. Wojciechowski, E. Bulska, J. Mieczkowski, K. Maksymiuk, Poly(n-butyl acrylate) based lead (II) selective electrodes, Talanta 79 (2009) 1247-1251.

[31] G. Lisak, E. Grygolowicz-Pawlak, M. Mazurkiewicz, E. Malinowska, T. Sokalski, J. Bobacka, A. Lewenstam, New polyacrylate-based lead(II) ion-selective electrodes, Microchim. Acta 164 (2009) 293-297.

[32] F. Sundfors, T. Lindfors, L. Höfler, R. Bereczki, R.E. Gyurcsányi, FTIR-ATR study of water uptake and diffusion through ion-selective membranes based on poly(acrylates) and silicone rubber, Anal. Chem. 81 (2009) 5925-5934. 